\title{
Luminosity of young Jupiters revisited
}

\section{Massive cores make hot planets}

\begin{abstract}
C. Mordasini ${ }^{\star}$
Max-Planck-Institut für Astronomie, Königstuhl 17, 69117 Heidelberg, Germany

e-mail: mordasini@mpia.de

Received 1 April 2013 / Accepted 22 June 2013

ABSTRACT

Context. The intrinsic luminosity of young Jupiters is of high interest for planet formation theory. It is an observable quantity that is determined by important physical mechanisms during formation, namely, the structure of the accretion shock and, even more fundamentally, the basic formation mechanism (core accretion or gravitational instability).

Aims. Our aim is to study the impact of the core mass on the post-formation entropy and luminosity of young giant planets forming via core accretion with a supercritical accretion shock that radiates all accretion shock energy (cold accretion).

Methods. For this, we conduct self-consistently coupled formation and evolution calculations of giant planets with masses between 1 and 12 Jovian masses and core masses between 20 and 120 Earth masses in the 1D spherically symmetric approximation.

Results. As the main result, it is found that the post-formation luminosity of massive giant planets is very sensitive to the core mass. An increase in the core mass by a factor 6 results in an increase in the post-formation luminosity of a 10-Jovian mass planet by a factor 120 , indicating a dependency as $M_{\text {core }}^{2-3}$. Due to this dependency, there is no single well-defined post-formation luminosity for core accretion, but a wide range, even for completely cold accretion. For massive cores ( 100 Earth masses), the post-formation luminosities of core accretion planets become so high that they approach those in the hot start scenario that is often associated with gravitational instability. For the mechanism to work, it is necessary that the solids are accreted before or during gas runaway accretion and that they sink during this time deep into the planet.

Conclusions. We make no claims about whether such massive cores can actually form in giant planets especially at large orbital distances. But if they can form, it becomes difficult to rule out core accretion as the formation mechanism based solely on luminosity for directly imaged planets that are more luminous than predicted for low core masses. Instead of invoking gravitational instability as the consequently necessary formation mode, the high luminosity can also be caused, at least in principle, simply by a more massive core.
\end{abstract}

Key words. planets and satellites: formation - planetary systems - planets and satellites: interiors

\section{Introduction}

Young self-luminous giant planets are important for planet formation theory because their luminosity still depends on important physical mechanisms occurring during the precedent formation phase. It has previously been understood (Marley et al. 2007, hereafter M07) that the structure of the accretion shock through which gas is accreted onto a planet has very important consequences for the post-formation luminosity. Inspired by the stellar case (Stahler et al. 1980), M07 showed for planets forming via core accretion that a supercritical shock radiating all liberated gravitational potential energy away leads to low post-formation entropies and luminosities (so-called cold accretion leading to a cold start, e.g., Hartmann et al. 1997; Baraffe et al. 2009).

These luminosities are so much lower than in conventional so-called hot start simulations beginning with an arbitrary high entropy state (e.g., Burrows et al. 1997; Baraffe et al. 2003) that the difference is easily observable with direct imaging observations. High entropy states are expected if the accretion shock is subcritical and does not radiate the shock luminosity (hot accretion), or if the gas is incorporated in the planet without going through a shock at all. This could happen if a massive patch of the protoplanetary disk becomes collectively

\footnotetext{
^ Reimar-Lüst Fellow of the MPG.
}

self-gravitationally unstable and directly forms a bound clump as envisioned in the gravitational instability formation model. The results of Galvagni et al. (2012) indicate that young giant planets formed by this mechanism indeed have high entropies.

One must, however, be careful in directly equating core accretion with cold start, and gravitational instability with hot start, as discussed in Mordasini et al. (2012a). In this work it was found that planets forming via the core accretion mechanism, but without radiative losses at the shock (hot accretion) have the same high post-formation entropies as are typical of hot starts. Either completely cold, or completely hot accretion are limiting assumptions that are solely chosen due to the current lack of knowledge about the actual shock structure in the planetary case (M07, see Commerçon et al. 2011, for the stellar first-core accretion shock). It is certainly reasonable to assume that the actual accretion onto a planet (both for core accretion and gravitational instability) is neither completely hot nor completely cold. This motivated Spiegel \& Burrows (2012, hereafter SB 12) to study a whole range of warm starts with intermediate entropies. Nevertheless, the potential difference in the luminosity of giant planets forming via the two different formation mechanisms explains why the luminosity of young planets is of strong interest for formation theory.

The ultimate goal must be to calculate the post-formation entropy for both mechanisms with $3 \mathrm{D}$ radiation-hydrodynamic 
simulation that resolve the accretion geometry onto the planet (presumably via a circumplanetary disk), the shock physics and the transport of radiation. In this work we consider in contrast the strongly idealized case of 1D spherically symmetric accretion. But we are nevertheless able to point out a new effect that potentially means that, even for completely cold accretion of the gas, there could be young giant planets formed via core accretion that are almost as hot as in the conventional hot start scenario.

The structure of the paper is as follows. In Sect. 2 we give an overview of the model and the settings used in the combined formation and evolution simulations that are presented in Sect. 3. The resulting post-formation entropies are shown in Sect. 4 where we present an updated version of the "entropy tuning fork". In Sect. 5 we show the luminosities during the evolution at constant mass after formation while Sect. 6 gives the radii during this phase. The conclusions are presented in Sect. 7. In Appendices A and B we give further information about the power-law approximation for the post-formation luminosity and the impact of several model settings on the core mass - initial entropy mapping, respectively.

\section{Model}

We simulate the formation and evolution of giant planets for different values of the initial surface density of planetesimals $\Sigma_{\mathrm{s}, 0}$ that translate into different final core masses. We use the planet formation code based on the core accretion paradigm initially presented in Alibert et al. (2004) that was recently extended into a self-consistently coupled formation and evolution code as shown in Mordasini et al. (2012b) where a detailed description can be found. The model solves a slightly simplified version of the standard equations describing the 1D (radial) internal structure of giant planets, consisting of the equations of mass conservation, hydrostatic equilibrium, energy conservation and energy transport (e.g., Bodenheimer \& Pollack 1986). The internal structure calculation is coupled with a model for the accretion rate of planetesimals as in Pollack et al. (1996) and the impact of planetesimals into the gaseous envelope (Podolak et al. 1988; Mordasini et al. 2006). In summary, the model allows us to make simulations similar to those of Fortney et al. (2005) or M07 that yield in particular the post-formation luminosity (i.e., the one after the final mass has been reached) of giant planets based selfconsistently on the precedent formation phase.

\subsection{Simulation setup}

We simulate the in situ formation and evolution of giant planets at 5.2 AU from a Sun-like star. Table 1 gives an overview of the most important settings and assumptions. Disk evolution and orbital migration are not included, but this should not alter the basic conclusions of the paper. The burning of deuterium is also neglected since it is not important at the maximal mass of $12 M_{4}$ (Jovian masses) that we consider (Spiegel et al. 2011; Mollière \& Mordasini 2012; Bodenheimer et al. 2013). The pressure and temperature in the protoplanetary disk are the same as in the J1 simulation of Pollack et al. (1996). The opacity of grains is reduced by a factor 0.003 relative to the ISM to approximate the consequences of grain evolution (Movshovitz et al. 2010). We do not model the hydrodynamic processes that govern the disklimited gas accretion rate in the runaway phase $\dot{M}_{X Y \text {, max }}$ but fix it to $0.01 M_{\oplus} / \mathrm{yr}$, a typical value (Lissauer et al. 2009). We consider final total masses between 1 and $12 M_{4}$, and vary the initial local surface density of planetesimals $\Sigma_{\mathrm{s}, 0}$ so that planets with different core masses $M_{\text {core }}$ form. The effect of planetesimal ejection is
Table 1. Settings for the formation and evolution calculation.

\begin{tabular}{lc}
\hline \hline Quantity & Value \\
\hline$a[\mathrm{AU}]$ & 5.2 \\
$\Sigma_{\mathrm{s}, 0}\left[\mathrm{~g} / \mathrm{cm}^{2}\right]$ & $10,12,15,18$ \\
$\dot{M}_{X Y, \max }\left[M_{\oplus} / \mathrm{yr}\right]$ & 0.01 \\
$T_{\text {neb }}[\mathrm{K}]$ & 150 \\
$P_{\text {neb }}\left[\mathrm{dyn} / \mathrm{cm}^{2}\right]$ & 0.275 \\
Dust to gas ratio & $1 / 70$ \\
Initial embryo mass $\left[M_{\oplus}\right]$ & 0.1 \\
Migration & not included \\
Disk evolution & not included \\
Planetesimal ejection & included \\
Core density & variable \\
Planetesimal size & 100 km \\
Fate of dissolved planetesimals & sink to core interface \\
Grain opacity red. factor $f_{\text {opa }}$ & 0.003 \\
Accretion shock luminosity & completely radiated \\
Atmosphere & gray approximation \\
Simulation duration & 20 Gyrs \\
\hline
\end{tabular}

included, and the density of the core is variable (Mordasini et al. 2012a). We assume that planetesimals (or their debris) always sink instantaneously to the core (discussion in Sect. 3.3).

During gas runaway accretion when the planet is detached from the nebula (so-called detached or transition stage cf. Bodenheimer et al. 2000), and the gas accretion rate is given by the disk-limited value, we consider two limiting cases for the shock structure: first, cold accretion where the complete accretion shock luminosity caused by the infall of the gas $L_{\text {acc }}$ is radiated away and does not contribute to the luminosity $L_{\text {int }}$ that is used to calculate the internal structure of the planet. The accretion shock luminosity is approximately given as $G M \dot{M}_{X Y} / R$ for a planet of mass $M$ and radius $R$ accreting gas at a rate $\dot{M}_{X Y}(G$ is the gravitational constant). Second we consider hot accretion without any radiative losses at the shock, so that the luminosity used to calculate the structure $L_{\text {int }}$ includes both $L_{\text {acc }}$ and the luminosity originating from the cooling and contraction of the material already incorporated in the planet.

The method of calculating this contribution and the temporal evolution of a planet is based on total energy conservation as described in Mordasini et al. (2012a). In this approach, the specific entropy of the newly accreted material $S_{\text {new }}$ is not an explicit input into the calculations. But a posteriori, it can be estimated by comparing the change in entropy in the convective zone during a time interval $\mathrm{d} t$, and the approximation that the entropy changes during this time only due to accretion of new gas, and not due to cooling of the material already in the planet. This holds approximately for cold $(S \lesssim 9.5$ in units of $k_{\mathrm{B}}$ per baryon as everywhere in this paper) high-mass planets $\left(M \gtrsim 3-5 M_{4}\right)$ where the Kelvin-Helmholtz timescale is much longer than the accretion timescale (see Sect. 3.2). Such cases have approximately horizontal entropy curves immediately after the end of accretion (bottom left panel of Fig. 1). For entropies $S$ in the convective zones at time $t$ and $t+\mathrm{d} t$, we then have $S(t+\mathrm{d} t) \approx f_{\text {new }} S_{\text {acc }}+\left(1-f_{\text {new }}\right) S(t)$, where $f_{\text {new }}$ is the mass fraction that has been accreted during $\mathrm{d} t,(M(t+\mathrm{d} t)-M(t)) / M(t+\mathrm{d} t)$. From these considerations, we find estimates (actually upper limits due to the neglect of cooling) for the difference in entropy between the newly accreted material and the material in the convective zone. One finds that the newly accreted material has an entropy that is lower by about 0.2 to $1.2 k_{\mathrm{B}}$ /baryon while a planets grows from 3 to $10 M_{4}$, with greater differences at higher masses, and initially smaller differences for lower core masses. 
C. Mordasini: Luminosity of young Jupiters revisited

\subsubsection{Boundary conditions}

During the detached stage, the outer boundary conditions for the internal structure are the same as in Mordasini et al. (2012a)

$$
\begin{aligned}
P & =P_{\mathrm{neb}}+\frac{\dot{M}_{X Y, \mathrm{max}}}{4 \pi R^{2}} v_{\mathrm{ff}}+\frac{2 g}{3 \kappa} & \tau & =\max \left(\rho_{\mathrm{neb}} \kappa_{\mathrm{neb}} R, 2 / 3\right) \\
T_{\mathrm{int}}^{4} & =\frac{3 \tau L_{\mathrm{int}}}{8 \pi \sigma R^{2}} & T^{4} & =(1-A) T_{\mathrm{neb}}^{4}+T_{\mathrm{int}}^{4} .
\end{aligned}
$$

The surface pressure $P$ thus contains the contribution from the nebula $P_{\text {neb }}$ (which quickly becomes negligibly low relative to the other terms), from the ram pressure ( $v_{\mathrm{ff}}$ is the free fall velocity), and from the photospheric pressure in the Eddington approximation ( $g$ is the gravitational acceleration on the planet's surface, $\kappa$ the Rosseland mean opacity). The expression for the optical depth $\tau$ includes the effect of the material surrounding the planet (Papaloizou \& Nelson 2005), but quickly becomes equal to $2 / 3$, because the radius $R$ decreases rapidly by roughly two orders of magnitude after detachment.

The surface temperature is given by the irradiation from the nebula with a temperature $T_{\text {neb }}$ assuming a Jupiter-like albedo $A$ and the contribution from the planet's own luminosity, $T_{\text {int }}$, which quickly after detachment becomes clearly dominant. As mentioned, $L_{\text {int }}$ does (does not) include $L_{\text {acc }}$ in the hot (cold) case.

These boundary conditions differ from the ones of Bodenheimer et al. (2000) and M07 in two aspects: first, they include the additional term due to the photospheric pressure. This is strictly speaking not self-consistent with a fully spherically symmetric geometry of the infall, because the photospheric pressure is derived under the assumption that the gas above the $\tau=2 / 3$ surface is in hydrostatic equilibrium, which is not the case in this geometry. On the other hand, in more complex geometries, the accreted material joins the planetary surface only in certain parts as suggested by 3D hydrodynamic simulations, namely through a circumplanetary disk (Tanigawa et al. 2012; Uribe et al. 2013) near (but maybe not exactly at) the midplane and/or polar regions (Ayliffe \& Bate 2012). In the regions without much gas accretion, the photospheric pressure would then (approximately) apply (Hartmann et al. 1997). We tested the impact of omitting the photospheric pressure on the post-formation entropy, finding rather small changes (see Appendix B).

The second difference in the boundary conditions relative to Bodenheimer et al. (2000) is related to the temperature. We simply assume that the material falling from the Hill sphere onto the planet is optically thin to the outgoing radiation. The model of Bodenheimer et al. (2000) is more refined, since they solve the radiative transfer equation in the diffusion approximation using a density profile given by the free fall and a constant opacity. They find that this leads to good agreement with more detailed solutions of the radiative transfer equation. It is clear that the detailed structure of the temperature of the infalling gas in front of the shock (e.g., concerning the radiative precursor, or the distinction between the optically thick or thin regime) is of high interest for more precise predictions about the planet's initial entropy.

To model this at least in 1D, it should be possible to use radiative hydrodynamic models similar to those of the accretion shock of the Larson's first core during star formation (e.g., Commerçon et al. 2011). At this stage of star formation, it is sufficient to use the grey approximation in the radiative transfer calculations (Vaytet et al. 2012) because one is dealing mostly with IR radiation. For the accretion shock on the (second) hydrostatic stellar core considered by Stahler et al. (1980), the temperatures become considerably higher, so that radiation over a
Table 2. Results for $10 M_{4}$.

\begin{tabular}{lccccc}
\hline \hline$\Sigma_{\mathrm{s}, 0}$ & 10 & 12 & 15 & 18 & $\mathrm{HS}$ \\
\hline$M_{\text {iso }}\left[M_{\oplus}\right]$ & 11.5 & 15.1 & 21.1 & 27.8 & 11.5 \\
$t_{\text {form }}[\mathrm{Myrs}]$ & 1.25 & 0.87 & 0.62 & 0.51 & 1.25 \\
$M_{\text {core }}\left[M_{\oplus}\right]$ & 32.1 & 38.9 & 48.9 & 58.6 & 35.1 \\
$S_{0}\left[k_{\mathrm{B}} / \mathrm{ba}.\right]$ & 9.09 & 9.31 & 9.63 & 9.90 & 13.40 \\
$R_{0}\left[R_{4}\right]$ & 1.31 & 1.36 & 1.44 & 1.52 & 4.94 \\
$L_{0}\left[L_{\odot}\right]$ & $5.1(-5)$ & $8.1(-5)$ & $1.5(-4)$ & $2.4(-4)$ & $1.2(-2)$ \\
$S_{\max }\left[k_{\mathrm{B}} / \mathrm{ba}.\right]$ & 11.03 & 11.15 & 11.29 & 11.41 & 13.40 \\
$L_{\mathrm{rga}}\left[L_{\odot}\right]$ & $6.5(-2)$ & $6.3(-2)$ & $6.0(-2)$ & $5.6(-2)$ & $1.2(-2)$ \\
\hline
\end{tabular}

Notes. For $L_{0}$ and $L_{\mathrm{rga}}$ the number in parentheses is the exponent $p$ in $10^{p}$.

wide frequency range (IR to X-rays) becomes important. As indicated by the calculations of Stahler et al. (1980), it is then necessary to consider a frequency-dependent treatment of the radiation. We note that the shock temperature $T_{\text {shock }}$ in the planetary case can in principle become quite high: for a supercritical shock (Commerçon et al. 2011)

$T_{\text {shock }}=\left(\frac{1 / 2 \rho_{\mathrm{ff}} v_{\mathrm{ff}}^{3}}{\sigma_{\mathrm{SB}}}\right)^{1 / 4}$

where $\rho_{\mathrm{ff}}=\dot{M}_{X Y} /\left(4 \pi R^{2} v_{\mathrm{ff}}\right)$ and $\sigma_{\mathrm{SB}}$ is the Stefan-Boltzmann constant. For a $10 M_{4}$ accreting at $10^{-2} M_{\oplus} / \mathrm{yr}$ in the cold case (radius only about $1.4 R_{4}$, see Table 2) $T_{\text {shock }} \approx 7600 \mathrm{~K}$, leading to important contribution from the UV to the IR domain, potentially making a frequency-dependent treatment necessary.

For the determination of the appropriate boundary conditions, the detailed description of the accretion shock itself is however not yet sufficient. Hartmann et al. (1997) study cold accretion onto fully convective low-mass stars, a situation closely related to the one here, at least before D ignition. They show that for accretion through a circumstellar disk, for large parts of the surface of the star, normal photospheric boundary conditions apply. This leads to a more efficient cooling compared to the spherical accretion geometry of Stahler et al. (1980), meaning that the global accretion geometry also matters. For giant planets, we may expect that initially, when the Hill sphere radius of the planet is still smaller than the disk's vertical scale height, the accretion is roughly spherically symmetric, while later on, the accretion occurs through a disk, with the later phase being dominant for massive planets (D'Angelo \& Lubow 2008). The gas in the circumplanetary disk would then eventually accrete into the planet via a viscous boundary layer or potentially magnetospheric accretion columns as for stars (Lovelace et al. 2011).

Once the desired total mass of a planet is approached in a simulation, the gas accretion rate is shut down on a short timescale. During the evolutionary phase at constant mass we use as Bodenheimer et al. (2000) a simple gray atmosphere with solar composition opacities from Freedman et al. (2008). This means that our results for the long term cooling are less accurate than those of models using non-gray models (e.g., Burrows et al. 1997; Baraffe et al. 2003).

\section{Combined formation and evolution simulations}

Using the model described in the last section, we simulate the formation and evolution of planets of different total and core masses with the goal to determine observable post-formation properties like luminosity and radius. Before we discuss the post-formation properties, we show the formation (and early 

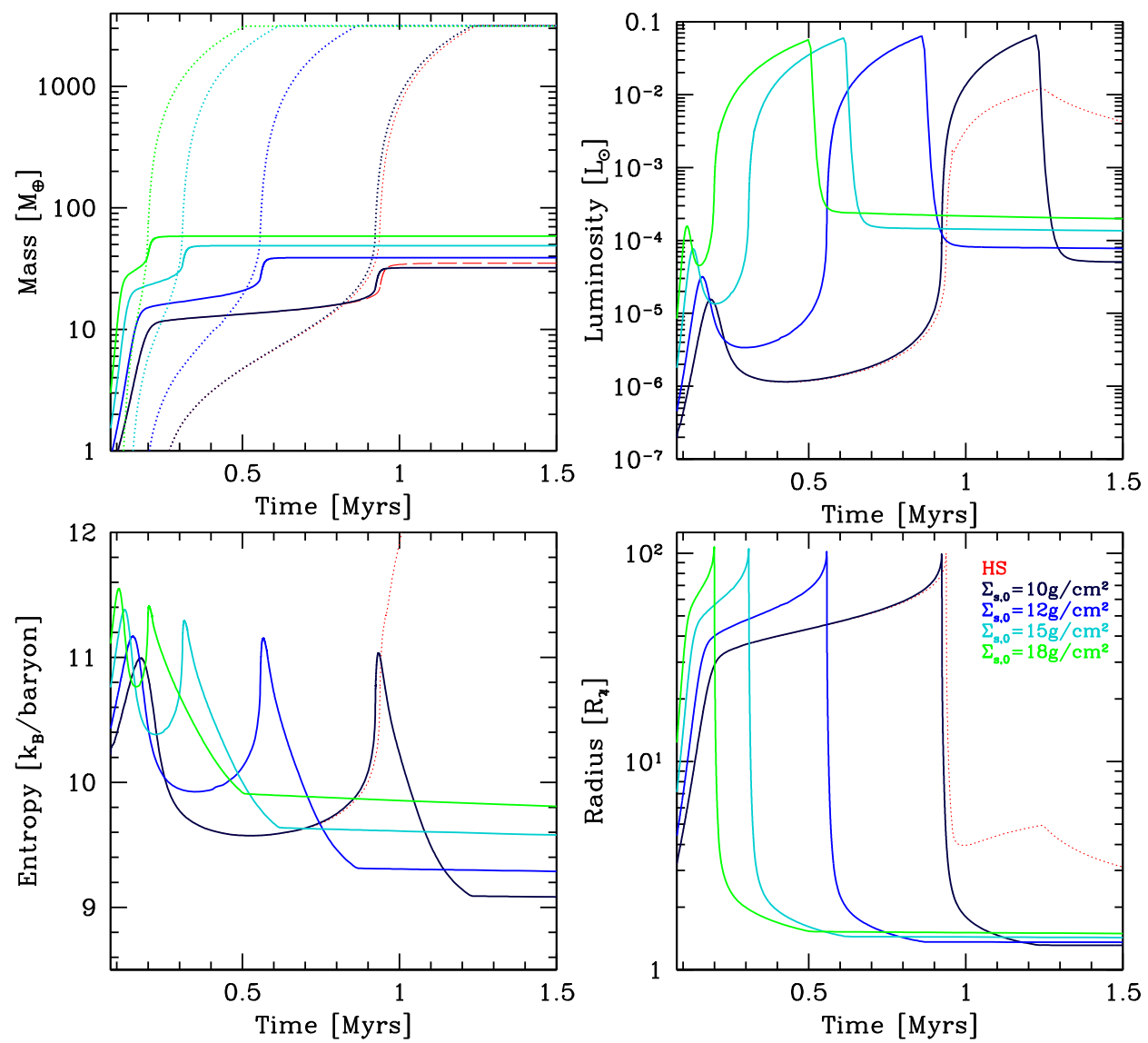

Fig. 1. Formation and early evolution of $10 M_{4}$ planets forming at 5.2 AU. The thin red line is for hot accretion (HS). The remaining four cases are all for cold accretion, but differ in the initial planetesimal surface density $\Sigma_{\mathrm{s}, 0}$ as indicated in the plot. The top left panel shows the core (solid) and total mass (dotted lines). The top right panel shows the total luminosity. The bottom left and right panels are the specific entropy at the core-envelope boundary, and the outer radius, respectively. Note how the luminosity, entropy and radius at the end of the formation phase increase with core mass for cold accretion.

evolution) of massive giant planets for different initial solid surface densities $\Sigma_{\mathrm{s}, 0}$.

\subsection{Formation of a $10 M_{4}$ planet for different $\Sigma_{s, 0}$}

Figure 1 shows the formation and early evolution of five $10 M_{4}$ planets. Four different initial solid surface densities of planetesimals $\Sigma_{\mathrm{s}, 0}$ are considered: 10 (nominal), 12,15 , and $18 \mathrm{~g} / \mathrm{cm}^{2}$. Four simulations are calculated under the cold accretion assumption, while an additional fifth comparison simulation also has $\Sigma_{\mathrm{s}, 0}=10 \mathrm{~g} / \mathrm{cm}^{2}$, but assumes hot accretion. The simulations are otherwise identical. The most important results of the simulations are listed in Table 2.

\subsubsection{Mass}

The top left panel shows the total mass $M$ and core mass $M_{\text {core }}$ as a function of time. The higher $\Sigma_{\mathrm{s}, 0}$, the shorter the formation time $t_{\text {form }}$ until the final mass of $12 M_{4}$ is reached, and the higher the final core mass, as expected (e.g., Pollack et al. 1996). The planets in this simulation continue to accrete planetesimals after crossover and in the runaway phase. This leads to a two step accretion of solids: first in phase $\mathrm{I}^{1}$, and then again

1 The phases I, II, and III refer to the main phases that are seen in classical in situ formation simulations of Jupiter, as defined in Pollack et al. (1996). During phase I the embryo accretes rapidly all planetesimals in the feeding zone, until the core reaches the isolation mass. The envelope mass is very low due to the high core luminosity. During phase II, a slow growth of the core and the gaseous envelope occurs where the planet has to accrete gas in order to expand its solid feeding zone. The accretion rate of gas is higher than the one of planetesimals. Phase III begins at the crossover point, i.e., the moment in time when the core at and after crossover due to the rapid extension of the feeding zone that is proportional to the Hill sphere radius $R_{\mathrm{H}}$. The final mass is then limited by the combined effects of planetesimal ejection and emptying of the feeding zone. During phase II and also during runaway, if the planet accretes all planetesimals in the feeding zone, it holds that $M_{\text {core }}=\left(M M_{\text {iso }}^{2}\right)^{1 / 3}$ (Pollack et al. 1996) where $M_{\text {iso }}$ is the isolation mass (Lissauer 1993). Since $M_{\text {iso }} \propto \Sigma_{\mathrm{s}, 0}^{3 / 2}$, this means that for a fixed $M$ the final core mass is proportional to $\Sigma_{\mathrm{s}, 0}$, which holds very well in the simulations (Table 2). The final core masses are between 32 and $59 M_{\oplus}$, i.e., they differ by a factor 1.8 as $\Sigma_{\mathrm{s}, 0}$. Regarding the mass, the hot accretion case is basically the same as the corresponding cold case, except for a slightly higher final core mass. It is the consequence of a larger capture radius due to an overall larger planet radius.

\subsubsection{Luminosity}

The top right panel shows the total luminosity, i.e., it includes for the cold accretion case both $L_{\text {int }}$ and $L_{\text {acc }}$. One sees the characteristic shape (e.g., Bodenheimer et al. 2000) with a first lower maximum during phase I that is due to the accretion of the core, then a low luminosity during phase II, and finally the gas runaway accretion phase where the planets are very bright. For cold accretion, the luminosity during this phase is increasingly dominated by $L_{\text {acc }}$ that is a factor $\sim 1000$ bigger than $L_{\text {int }}$ at the moment the

and envelope mass become equal. Afterwards, a rapid increase of the gas accretion rate occurs, leading to runaway gas accretion. Note that it is far from clear that these phases actually occur: Phase I is only possible if the random velocities of the planetesimals stay low, which is clearly questionable for large planetesimals (e.g., Ida \& Makino 1993). Phase II can be suppressed by orbital migration (Alibert et al. 2004) or the opening of a gap in the planetesimal disk (Shiraishi \& Ida 2008). 
gas accretion rate is artificially reduced because a mass of $10 \mathrm{M}_{4}$ is approached. The overall highest luminosity is reached just before the reduction of the gas accretion begins $\left(L_{\mathrm{rga}}\right)$. While difficult to see on the logarithmic scale, the $L_{\text {rga }}$ for the planet with the most massive core is actually about $15 \%$ lower than in the simulation with the lowest core mass. This difference is relevant, as will become clear below. During the reduction of $\dot{M}_{X Y}$, the luminosity in the cold accretion simulations falls sharply since the contribution of $L_{\text {acc }}$ vanishes. Once the final mass has been reached, the luminosity takes the value of the intrinsic luminosity at the end of formation respectively at the beginning of evolution, $L_{0}$. The central result of this paper is now that this $L_{0}$ is not identical in the four cold accretion simulations. Instead, it is a strong function of the core mass: $L_{0}$ is $5.1 \times 10^{-5} L_{\odot}$ for $M_{\text {core }}=32 M_{\oplus}$, but $2.4 \times 10^{-4} L_{\odot}$ for $M_{\text {core }}=59 M_{\oplus}$. The increase of $M_{\text {core }}$ (and $\Sigma_{\mathrm{s}, 0}$ ) by a factor 1.8 has thus caused an increase of $L_{0}$ by a factor 4.7 , which corresponds for this interval in core mass to a power-law dependence roughly like $M_{\text {core }}^{2.6}$ (see Appendix A for a discussion of the power-law approximation). The origin of this effect is discussed in Sect. 3.2. As we will see further down in Sect. 5, the effect is strong enough to have potentially observable consequences.

\subsubsection{Entropy}

The bottom left panel shows the entropy $S$ at the core-envelope interface. As expected from the luminosity curve, there are two maxima, one during phase I, and a second one shortly after detachment $\left(S_{\max }\right.$, cf. Table 2$)$. The planets detach from the disk at a mass of about $100 M_{\oplus}$ (slightly increasing with $\Sigma_{\mathrm{s}, 0}$ ), and then contract initially very rapidly. The maximal entropy is reached when the outer radius has decreased to about 6-10 $R_{4}$ (Jovian radii), and the mass is around 150 to $200 M_{\oplus}$. After this maximum that is higher for more massive cores, the entropy steadily decreases due to the addition of low-entropy material for cold accretion. Note that the four entropy curves diverge during this decrease, i.e., the relative differences in $S$ between the four simulation increases. At the end of formation, there is a difference in the entropies $S_{0}$ of 0.81 . For hot accretion, the entropy in contrast increases during the entire runaway phase to reach a maximum value $S_{\max }=S_{0}=13.31$ just before accretion is terminated (outside of the figure).

\subsubsection{Radius}

The bottom right panel shows the outer radius. The basic shape of the curves follows the pattern found by Bodenheimer et al. (2000) with a large radius during the attached phase $\left(\sim R_{\mathrm{H}}\right)$, then the rapid collapse, and finally the slower contraction once the interior becomes sufficiently degenerate. We see that the planet with the most massive core has the largest radius $R_{0}$ at the end of the formation epoch. Its higher entropy leading to a larger radius (e.g., SB 12) thus dominates at least initially over the effect that a higher heavy element fraction reduces the radius (e.g., Fortney et al. 2007; Baraffe et al. 2008). As the planets cool, the radii decrease and the memory of the initially higher entropy gets lost, so that at about $120 \mathrm{Myrs}$, the radius of the planet with the most massive core and the one of the planet with the lowest core mass cross over, and afterwards, the planet with the more massive core has a smaller radius, as it is normally the case (see Sect. 6).

For cold accretion, the planets' radii decrease all the time during runaway accretion while they grow in mass. For hot accretion, the planet in contrast first collapses rapidly to a radius of about $4 R_{4}$ (when $M \approx 2 M_{4}$ ) and then re-inflates to $R_{0}=$ $4.94 R_{4}$ at the end of formation due to the addition of highentropy material. This $R_{0}$ is similar to the "initial" radius assumed in evolutionary hot start simulations like, e.g., SB 12 who use $R_{0}=4.15 R_{4}$ (the "initial" radius is of course ill-defined due to the extremely short $\tau_{\mathrm{KH}}$ ). This agreement indicates that our simplification for the solution of the internal structure equations (constant luminosity as a function of radius) does not lead to strong departures from the typical hot start scenario, despite the complications (absence of a deep radiative zone) the $\partial L / \partial r=0$ approximation could potentially have for hot accretion (see Mordasini et al. 2012b).

\subsection{Self-amplifying process}

One may wonder how a change in the core mass by a small fraction of the planet's total mass $\left(27 M_{\oplus}\right.$ or just $0.8 \%$ of the total mass) can significantly affect the luminosity of a $10 M_{4}$ planet. The reason is a self-amplifying process during gas runaway accretion, combined with the fact that for a higher $\Sigma_{\mathrm{s}, 0}$, the entropy is already higher when runaway gas accretion starts. The latter is due to the fact that for a higher $\Sigma_{\mathrm{s}, 0}$, a more massive core (and envelope) forms on a shorter timescale, which means that the luminosity must be higher and so the entropy. This leads to a $S_{\max }$ that is a factor 1.03 higher in the simulation with the most massive core relative to the nominal case. A consequence of the higher entropy at detachment is that the outer radius of the high $M_{\text {core }}$ planet is larger for a given mass. This means that the gas that is accreted during runaway falls less deep in the potential well of the planet, so the shock is weaker and $L_{\mathrm{acc}} \approx G M \dot{M}_{X Y} / R$ is lower. Less liberated potential energy is thus radiated, instead the gas is incorporated at a higher entropy into the planet. The higher entropy causes a larger radius, which again means a weaker shock and so on, i.e., there is a self-amplifying process. This means that the relative difference in entropy between the different $M_{\text {core }}$ cases continuously increases in the detached phase. Indeed, the ratio of the entropy $S_{0}$ in the planet with the most massive core relative to the nominal case has increased to 1.09 at the end of formation. Due to the strong sensitivity of the luminosity on $S$ (e.g., Marleau \& Cumming 2013) such factors matter.

At the same mass, a planet with a higher entropy would usually contract faster because the Kelvin-Helmholtz timescale $\tau_{\mathrm{KH}}$ is shorter. This would stop the self-amplifying process. But for the assumed gas accretion rate in runaway of $\dot{M}_{X Y \text {, max }}=$ $10^{-2} M_{\oplus} / \mathrm{yr}$, the accretion timescale $\tau_{\text {acc }}=M / \dot{M}_{X Y \text {, max }}$ is most of the time shorter than $\tau_{\mathrm{KH}}$ (calculated as $\left|E_{\text {tot }}\right| / L_{\text {int }} \sim G M^{2} /\left(R L_{\text {int }}\right)$ where $E_{\text {tot }}$ is the sum of the gravitational and thermal energy in the planet). Early after detachment, approximately at the same time when $S_{\max }$ occurs, $\tau_{\mathrm{KH}} / \tau_{\text {acc }}$ first falls slightly below unity, and the radii of the planets indeed start to converge. But the radius of the planet with the more massive core remains still larger, and with increasing mass, the ratio $\tau_{\mathrm{KH}} / \tau_{\text {acc }} \propto M /\left(R L_{\text {int }}\right)$ increases ( $L_{\text {int }}$ and $R$ decrease during the runaway accretion phase for $M \gtrsim 300 M_{\oplus}$ for cold accretion). Just before the gas accretion is ramped down, $\tau_{\mathrm{KH}} / \tau_{\mathrm{acc}} \approx 600$ and 100 , for $M_{\text {core }}=32$ and $59 M_{\oplus}$, respectively. This means that the planet with the higher core mass cannot sufficiently rapidly contract to stop the selfamplification. Instead, the initially relatively low entropy difference gets amplified, with the described consequences for the post-formation luminosity.

This mechanism also explains why $L_{\mathrm{rga}}$ is $15 \%$ lower in the simulation with the highest core mass and cold accretion than in the nominal case. For hot accretion $L_{\text {rga }}$ (that is here the same 
as $L_{0}$ ) is even a factor 5.4 lower than in the nominal cold accretion simulation. The lower the luminosity during gas runaway accretion, the higher the luminosity during evolution, because the planet stores via a higher entropy the part of the gravitational potential energy that is not radiated away during formation. While this fundamental mechanism is relatively general, it is clear that the quantitative consequences (specific values of $S_{0}$ ) depend on assumptions like the gas accretion rate during runaway (that is certainly not constant, cf. Lissauer et al. 2009; Bodenheimer et al. 2013) or the infall geometry that is certainly not spherically symmetric (e.g., Ayliffe \& Bate 2009) (see also Appendix B).

\subsection{Sinking approximation}

An important assumption necessary for the mechanism to work efficiently is that the planetesimals or their debris sink to the core (or at least deep into the planet) on a short timescale $\left(\ll \tau_{\mathrm{acc}}\right)$, so that they liberate a significant amount of potential energy to heat the planet during the formation phase. Especially for the planetesimals accreted after crossover (i.e., during the second phase where the core grows significantly, see Sect. 3.1.1) it is unclear whether the sinking approximation holds. This is because the gaseous envelope at this moment is already hot and massive (several $10 M_{\oplus}$ ) while simulations of the impacts of planetesimals (Podolak et al. 1988; Mordasini et al. 2006) into protoplanetary atmospheres rather indicate that $100 \mathrm{~km}$ planetesimals can only directly penetrate through envelopes with masses of a few $M_{\oplus}$ (Mordasini et al. 2006). In more massive envelopes, the planetesimals get fragmented and then vaporized by shock wave radiation during the impact. For this material to also contribute efficiently to the heating of the planet, the planetesimal debris has to sink after the impact. Iaroslavitz \& Podolak (2007) studied this by always comparing the partial and the saturation vapor pressure of the high- $Z$ material in all layers during a formation simulation of Hubickyj et al. (2005) so that they can track where the high- $Z$ material ends up. They find that even if much high$Z$ material does not reach the core in solid form directly, it is still concentrated (as vapor) in the envelope layers closely above it. They therefore conclude that the gravitational energy release (which is crucial in this study) is similar to the sinking approximation we use here. The study of Iaroslavitz \& Podolak (2007) is simplified in a number of aspects and only follows the evolution of the debris up to the crossover mass, when core and envelope mass are equal. The fate of planetesimal material accreted after this point is thus less clear. For this material also to sink, it would maybe be necessary that an equivalent mechanism occurs as for the heating of giant planets by He-separation (e.g., Stevenson \& Salpeter 1977): the metals would need to undergo de-mixing from the H/He (e.g., Wilson \& Militzer 2012), to nucleate and to grow into sufficiently large droplets in order to settle despite convention, and this on a sufficiently short timescale (Li et al. 2010). Whether this is possible needs to be checked in future work.

In order to test if it is important at which moment in time the planetesimals are accreted (during phase I or also after crossover), we conducted simulations where the accretion of planetesimals is artificially shut down once crossover is reached, as it seems to be the case in the simulations of M07. At this moment the core mass is approximately equal to $\sqrt{2} M_{\text {iso }}$ (Pollack et al. 1996). We then adjusted the initial surface density of planetesimals in a way that this mass is the same as the final core mass in the normal simulations where the planets continue to accrete planetesimals also after crossover. We find that

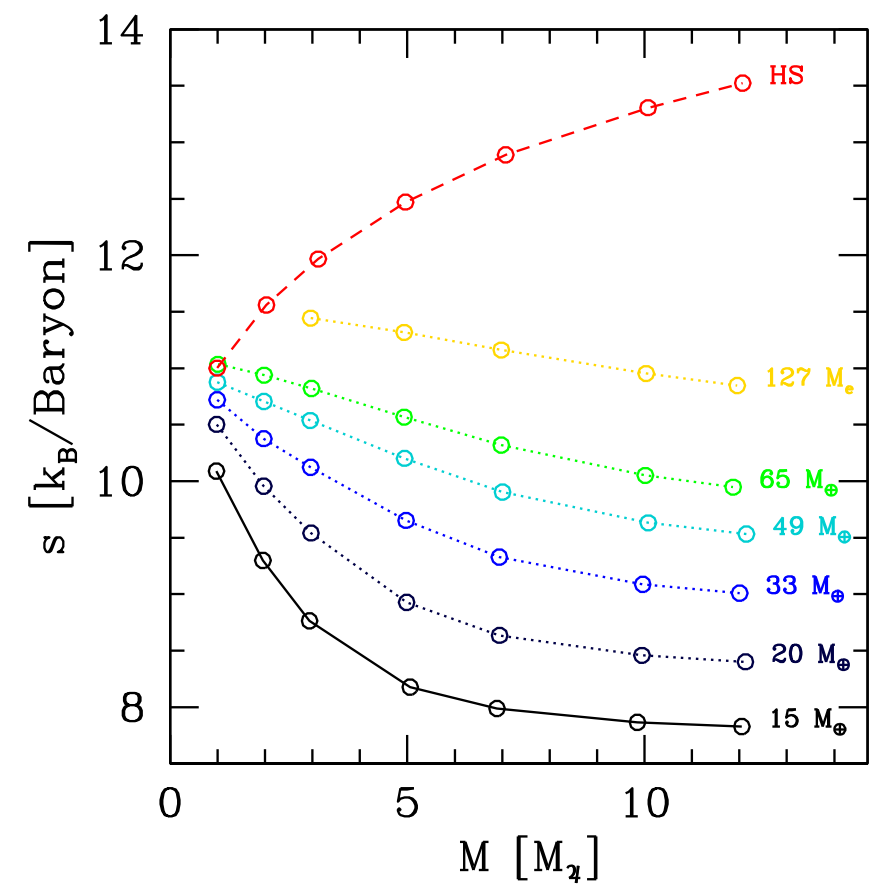

Fig. 2. Post-formation entropy as function of total mass and for six different core masses indicated in the plot for cold accretion. The red dashed line labelled HS is for hot accretion.

the resulting post-formation luminosities are very similar. This means that for the core mass to influence the luminosity after formation, it is not necessary that the massive core forms during the runaway phase when it is, as explained, potentially more difficult for the solids to penetrate deep enough. It is sufficient that a massive core forms at all, especially during phase I and before crossover, when it seems that the sinking approximation applies at least in an approximate way. But it is clear that to reach the same core mass without accretion during runaway, a higher $\Sigma_{\mathrm{s}, 0}$ (approximately by a factor 1.5 to 2 ) is needed.

Note that in particular in the absence of sinking, a sufficiently high energy input due to impacts can lead to the formation of a deep radiative zone ( $\mathrm{Li}$ et al. 2010), a process similar to the formation of a radiative zone during hot accretion onto an initially fully convective star (Mercer-Smith et al. 1984; Prialnik \& Livio 1985; Hartmann et al. 1997). For sufficiently high accretion rates, the stars inflate, which could in principle also lead to a weaker accretion shock. This will also be addressed in future work.

\section{Post-formation entropy as function of total and core mass}

We have conducted combined formation and evolution simulations as in the last section but for total final masses between 1 and $12 M_{4}$, and $\Sigma_{\mathrm{s}, 0}$ leading to final core masses between approximately 15 and $127 M_{\oplus}$. Figure 2 shows the resulting specific entropies at the core-envelope boundary. The entropies $S_{0}$ are shown immediately after the moment when a planet has reached its final mass, i.e., when the evolutionary phase begins.

One sees the characteristic "entropy tuning-fork" (M07; SB 12; Marleau \& Cumming 2013). For cold accretion, the entropy decreases with increasing mass since an increasingly large fraction of the planet's mass is accreted through the entropy reducing shock, while for hot accretion, it increases because an increasingly large amount of liberated potential energy is stored in 
the planets. The new result is that the level of the cold branch of the fork increases with increasing core mass. For massive planets, the entropy is about $3 k_{\mathrm{B}}$ /baryon higher for the most massive core we have considered $\left(127 M_{\oplus}\right)$ than for the lowest value of $15 M_{\oplus}$. At a total mass of $12 M_{4}$, this is more than half of the difference between the $15 M_{\oplus}$ core cold case and the hot accretion case, i.e., this is a great difference. We thus find that the mass of the core leads to a wide continuum of intermediate warm starts (SB 12) for core accretion planets. While SB 12 envisioned these warm starts to be the consequence of intermediate radiative efficiencies of the accretion shock (which is a perfectly possible alternative explanation) we find that even for a fully efficient shock, there is a wide range of post-formation entropies if planets can have high (but not exorbitant) core masses.

It is interesting to compare our results with those of M07. One has to take into account that we show the entropy at $t-t_{\text {form }}=0$ Myrs, whereas M07 show it after 1 Myr of evolution which matters for the low-mass planets because of their short $\mathrm{KH}$ timescale. We find that for cold accretion, a $1 M_{4}$ planet has at $1 \mathrm{Myr}$ an entropy of 9.30 and 9.36 for core masses of 15 and $20 M_{\oplus}$, respectively, while M07 found 9.25 for a core mass of $17 M_{\oplus}$. The $10 M_{4}$ planet in our simulations has at $1 \mathrm{Myr}$ an entropy of 7.86 and 8.45 for core masses of 15 and $20 M_{\oplus}$ (virtually identical as at the beginning), which brackets the 8.25 found by M07 for a $19 M_{\oplus}$ core. There is thus good agreement between the two completely independent codes despite the multitude of assumptions that enter into them. For hot accretion, the differences are somewhat greater, as our planets have at $1 \mathrm{Myr}$ entropies that are 0.2 to 0.4 lower. But this is not surprising, because M07 uses arbitrary initial entropies for the hot case, which still matter after 1 Myr of evolution.

Regarding the absolute values of the post-formation entropy, we need however to keep in mind that both codes are 1D. Multidimensional hydrodynamic simulations point in contrast to complex accretion geometries (Tanigawa et al. 2012; Ayliffe \& Bate 2012; Uribe et al. 2013). This means that the quantitative results found here, and in particular the specific core mass - initial entropy mapping should not be taken as final results. Rather, they are indicators of possible important mechanism and correlations. They should be reassessed and re-quantified as soon as multidimensional, (magneto-)radiation hydrodynamic simulations with sufficiently high resolution and a realistic equation of state can directly predict the initial entropy of planets. In Appendix B we quantify the impact of some model settings on the initial entropy within our 1D model framework.

We finally note that for hot accretion, the mass of the core is not important: for an increase of the core mass from 35 to $132 M_{\oplus}$ the post-formation entropy of a $10 M_{4}$ planet grows by just 0.1 , more than 20 times less than in the cold case. This is expected, because the accretion of gas represents for a radiatively inefficient shock a much stronger heating source than the accretion even of a massive core, and the self-amplifying mechanism described above cannot operate either.

\section{Luminosities during evolution}

Figure 3 shows the luminosity of giant planets for different total and core masses as a function of time after the final mass has been reached. The plot shows the results for cold accretion with four different core masses as well as for hot accretion. It additionally depicts the measured luminosities of seven young giant planets. Note that the total and core masses of the model planets can vary slightly around the values indicated in the plots, since the masses are a product of the formation phase.
The basic result is the same as in earlier studies (M07): cold accretion leads to fainter planets, and the difference is greater and remains longer for more massive planets. This is due to the greater differences in the post-formation entropies, and the longer Kelvin-Helmholtz timescale of more massive planets. The plot shows that quantitatively, however, the magnitude of the difference between cold and hot accretion strongly depends on the mass of the core. The higher the core mass, the closer the cold and hot accretion cooling tracks. The impact of $M_{\text {core }}$ increases with total mass. For a $10 M_{4}$ planet at $1 \mathrm{Myr}$, for example, the luminosity with the most massive core is a factor $\sim 100$ higher compared to the lowest core mass, and therefore only a factor 2 to 3 lower than for hot accretion.

The much higher post-formation entropy of high $M_{\text {core }}$ planets also strongly reduces the time until cold and hot accretion cooling curves converge, due to the exponential dependency of the $\mathrm{KH}$ timescale on $S$ (e.g., M07). At a core mass of $20 M_{\oplus}$, it takes of order $10^{9} \mathrm{yrs}$ until the luminosities of a $10 M_{4}$ planet become similar for cold and hot accretion, as already found by M07, whereas at $M_{\text {core }}=127 M_{\oplus}$, it just takes $\sim 10^{7}$ yrs.

The plot also shows the luminosities of 1RXS1609 b (Lafrenière et al. 2008), $\beta$ Pic b (Lagrange et al. 2010), 2M1207 b (Chauvin et al. 2004), and the HR 8799 planets (Marois et al. 2008) as calculated by Bonnefoy et al. (2013). While we do not make any claim that our models are specifically applicable to any particular object, the comparison of these objects with the cold accretion cooling tracks makes the importance of $M_{\text {core }}$ clear. At $M_{\text {core }}=20 M_{\oplus}$, cold accretion is not consistent with any observation because the planets are too faint (as previously noted in several works), while for a core roughly 6 times more massive, all observed luminosities can be reproduced, and the mass estimates are virtually identical as for hot accretion for the less bright planets, and less than $2 M_{4}$ higher for the brighter ones.

The dependency on $M_{\text {core }}$ makes the conversion of luminosity into mass for cold accretion difficult: for HR 8799 b, for example, one deduces a mass clearly exceeding $12 M_{4}$ for $M_{\text {core }}=$ $20 M_{\oplus}$, but masses of $\sim 12,9$, and $7 M_{4}$ for $M_{\text {core }}=33,49$, and $127 M_{\oplus}$, the latter value being identical with hot accretion.

\section{Radii during evolution}

Figure 4 shows the radii of the planets as a function of total mass for different core masses at six moments in time relative to the end of the formation phase. Immediately after the end of formation (which is for a fixed gas accretion rate at a later absolute moment in time for more massive planets), there is a relatively simple situation. The planets formed with hot accretion (core mass of $\approx 35 M_{\oplus}$ ) have large radii that increase with increasing mass, well separated from the cold accretion planets. The initial radius of a $12 M_{4}$ planet is quite large $\left(\approx 5 R_{4}\right)$, and also a $1 M_{4}$ planet is at about $3.2 R_{4}$. The planets formed with cold accretion in contrast all have radii that decrease with increasing mass, a consequence of an increasingly large fraction of the planet's total mass having passed through the entropy-reducing shock. The higher the core mass, the larger the radius, a consequence of the higher entropy for more massive cores, as we had seen in the exemplary simulations (Sect. 3.1.4). The higher entropy thus dominates at this time over the well-known mechanism that a higher heavy element fraction normally leads to a smaller total radius (e.g., Fortney et al. 2007; Baraffe et al. 2008).

The further evolution is governed by the interplay of the different entropy states, the different core masses, and the different Kelvin-Helmholtz timescales that are very short for hot 

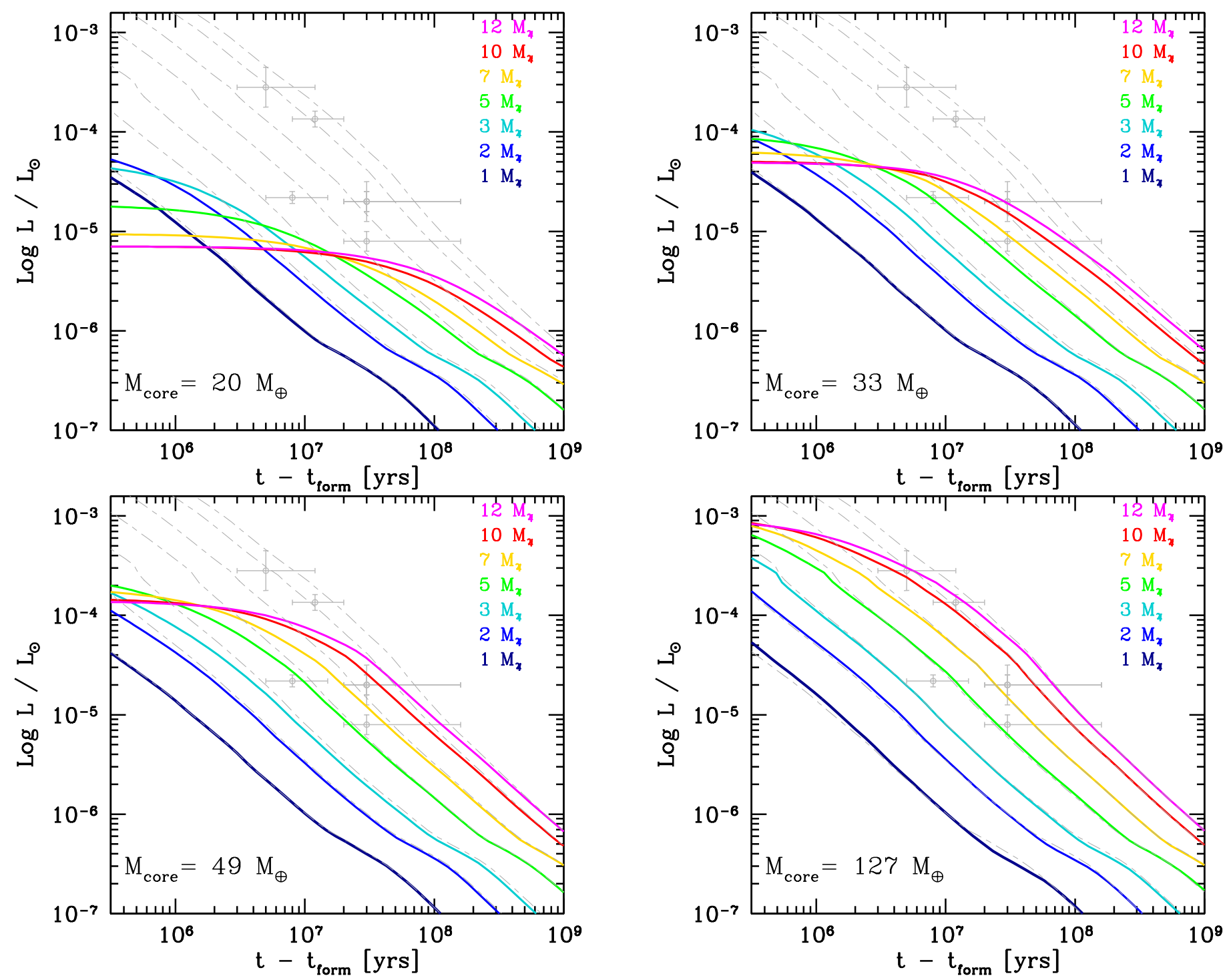

Fig. 3. Luminosity as a function of time after formation for planets with total masses of 1, 2, 3, 5, 7, 10, and $12 M_{4}$ and core masses of 20, 33, 49, and $127 M_{\oplus}$ as indicated in the panels. The colored solid lines assume cold accretion. The dashed-dotted gray lines assume hot accretion. The points with error bars are young giant planets (from top to bottom: 1RXS1609 b, Beta Pic b, 2M1207 b, HR8799c,d,e, HR8799 b).

(high $M_{\text {core }}$ ) low-mass planets, but very long for cold (low $M_{\text {core }}$ ) massive planets. The KH timescale sets the time after which the planets have forgotten the initial conditions (M07). At 1 Myrs, the curves for the $1 M_{4}$ planet have already crossed over, so that the planet with the most massive core now has the smallest radius, clearly a consequence of the very short $\tau_{\mathrm{KH}}$ of this planet $\left(\sim 10^{5} \mathrm{yrs}\right)$. For increasingly massive planets, it takes increasingly long until cross over occurs and the "normal" sequence of radii as a function of core mass is established. For the $12 M_{4}$ planet, e.g., cross over only happens at about 1 Gyr. At 10 Myrs, the "normal" order has been established also for $2 M_{4}$ planets, while the radii for all $3 M_{4}$ planets are nearly equal with the exception of the planet with the most massive core. The radii of the hot accretion planets have significantly decreased by now, so that there is nearly a continuum of radii going at $12 M_{4}$ from 1.1 to $1.5 R_{4}$ that includes the cold and hot accretion planets. At 100 Myrs, another feature becomes visible, namely the wellknown local maximum in the planetary mass-radius relationship (Zapolsky \& Salpeter 1969) at about $4 M_{4}$ that is due to the increasing degeneracy in the interior (see, e.g., Chabrier et al. 2009 , for a review). This feature becomes even more visible at
1 Gyr. At $12 M_{4}$, the radius of the $127 M_{\oplus}$ core planet is now approximately equal to the one of the $15 M_{\oplus}$ core planet. To reach this state, the former planet has contracted by about $0.9 R_{4}$ since formation, while the latter has only shrunk by a minuscule $0.02 R_{4}$. At $10 \mathrm{Gyrs}$, the direct influence of the core to reduce the radius eventually dominates at all masses over its influence during formation to cause larger radii via a higher entropy, so that the "normal" sequence of radii is established everywhere. The impact of the core mass is higher for planets with a lower total mass, as expected (e.g., Fortney et al. 2007).

\section{Conclusions}

The post-formation luminosity of giant planets forming via core accretion with a radiatively efficient shock depends significantly on the mass of the core, roughly like $M_{\text {core }}^{2-3}$. This dependency means that there is no single well-defined luminosity associated with core accretion even if all shock luminosity is radiated, but that there is a continuum of post-formation states (entropies, radii, and luminosities). The states range from cold states for core masses of $\sim 15 M_{\oplus}$ as in M07, over intermediate warm states 
C. Mordasini: Luminosity of young Jupiters revisited
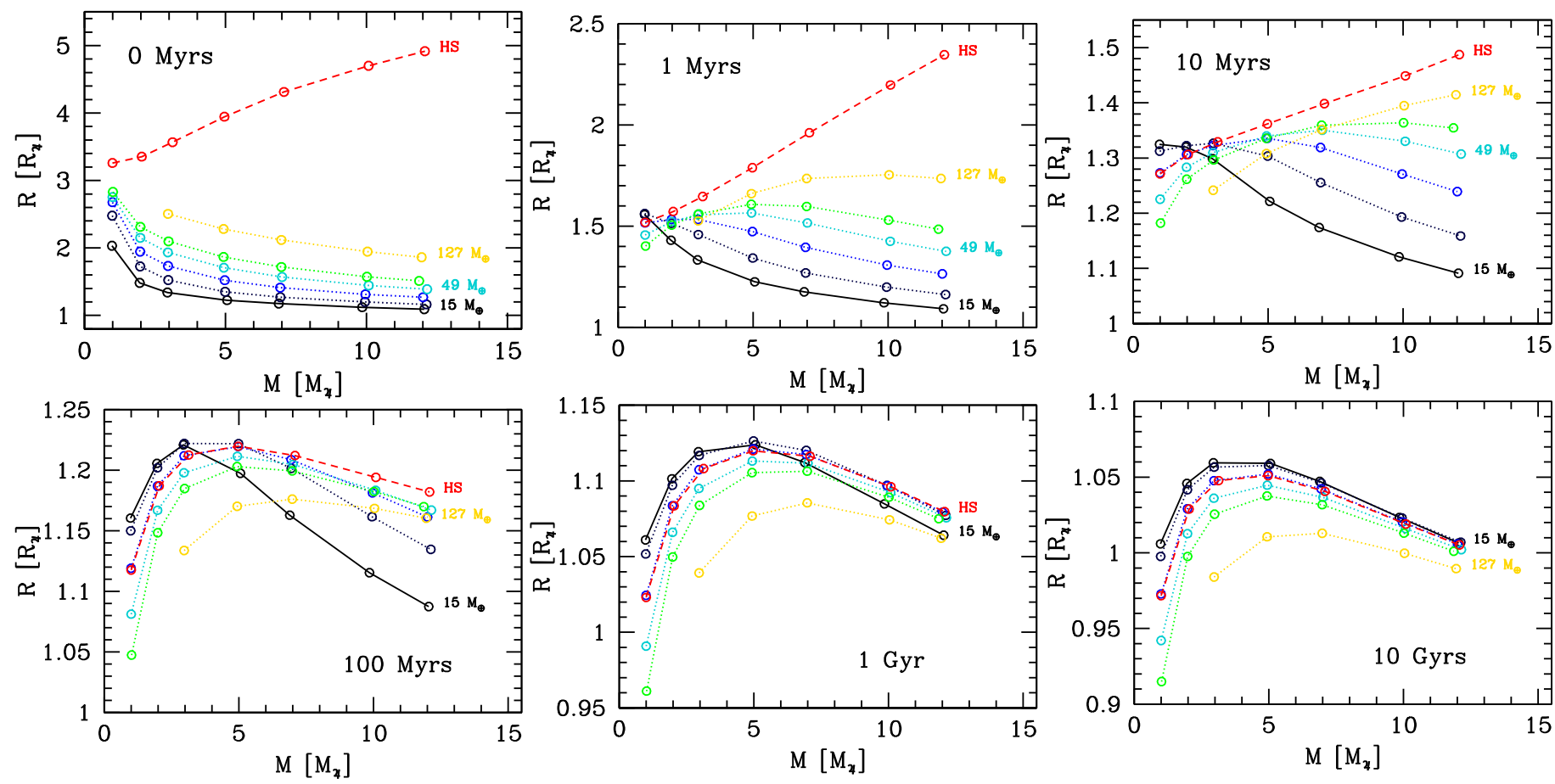

Fig. 4. Radii of planets as a function of mass between 1 and $12 M_{4}$ for different core masses at six different moments in time (relative to the end of accretion) as indicated in the panels. The core masses that are also partially indicated in the panels and the associated colors are the same as in Fig. 2. HS stands again for hot accretion.

(SB 12) to states that approach the classical hot state at core masses exceeding $100 M_{\oplus}$. The timescale until the luminosity of hot and cold accretion planets agree is strongly reduced for massive cores.

Internal structure models of transiting giant exoplanets (e.g., Guillot et al. 2006; Burrows et al. 2007; Baraffe et al. 2008; Miller \& Fortney 2011) indicate high amounts of heavy elements $\left(\sim 100 M_{\oplus}\right)$ to be present in several planets, and some planets (e.g., CoRoT-20b, Deleuil et al. 2012), even seem to contain 300-1000 $M_{\oplus}$. It is unknown where these solids reside inside the planets (concentrated in the core or mixed through the envelope) and how/when they were accreted. For the influence of planetesimal accretion on the post-formation luminosity to be strong, it is necessary that (1) the solids are accreted in high amounts during or before runaway gas accretion; and (2) that they sink deep into the planet during this period. In this paper, we do not make any claims about whether or not this is possible for an, e.g., $100 M_{\oplus}$ core in particular if the planet forms at a large orbital distance ( $\gg 10$ AU) where the accretion timescale at least for $\mathrm{km}$-sized planetesimals is long. We note that for smaller objects this seems to be significantly different, since aerodynamically assisted accretion rates of boulders or pebbles can be very high also in the outer disk (e.g., Ormel \& Klahr 2010; Lambrechts \& Johansen 2012). In any case, future studies must investigate both requirements based on solid accretion rates and the fate of solids inside the planet (cf. Iaroslavitz \& Podolak 2007). We just point out that if massive cores can indeed form, then their impact on the luminosity is high, and this already for a more "normal" core mass of $30 M_{\oplus}$.

A consequence of our results is that it becomes difficult to rule out core accretion as the formation mechanism based solely on luminosity for directly imaged planets that are more luminous than predicted by M07 (who considered $M_{\text {core }} \leq 19 M_{\oplus}$ ) or the equivalent simulations here for low core masses. Instead of invoking gravitational instability as the consequently necessary formation mechanism, the high luminosity could also be caused, at least in principle, by more massive cores in some of the planets. Such a more massive core has similar consequences as an accretion shock that does not radiate all accretion shock luminosity (SB 12), meaning that there is a degeneracy between the two mechanism (only at later times, the two become distinguishable through a smaller total radius of a planet with a more massive core).

In summary we see that concerning the luminosity of young Jupiters, we face a situation that is more complex than previously thought. The formation mechanism, the shock structure, but also the core mass play an important role. The atmospheric composition measured with multi-band photometry or spectroscopy (e.g., Janson et al. 2010; Bonnefoy et al. 2013; Konopacky et al. 2013) could be helpful in this situation to distinguish the different scenarios, because it seems likely that the presence of a massive core is associated with an enhanced metal content of the atmosphere.

Acknowledgements. I thank Hubert Klahr, Paul Mollière, Yann Alibert, Willy Benz, Mickaël Bonnefoy, Thomas Henning and Kai-Martin Dittkrist for useful discussions, and the Max-Planck-Gesellschaft for the Reimar-Lüst Fellowship. Computations were made on the BATCHELOR cluster at MPIA. I thank the referee Dr. David Spiegel for a constructive review. During the preparation of this work, we became aware that Bodenheimer et al. (2013) independently also found the dependency of the post-formation entropy on the core mass in another context (deuterium burning). For a $12 M_{4}$ planet and in the overlapping core mass range of 15 to $35 M_{\oplus}$, the two works predict post-formation entropies as function of the core mass that agree well, namely to about $0.2 k_{\mathrm{B}}$ /baryon (Fig. 11 in Bodenheimer et al. 2013 and Fig. 2 of this work).

\section{Appendix A: Power-law approximation for the post-formation luminosity}

In Sect. 3.1.2 an approximative power-law dependency of the post-formation luminosity on the core mass was mentioned 


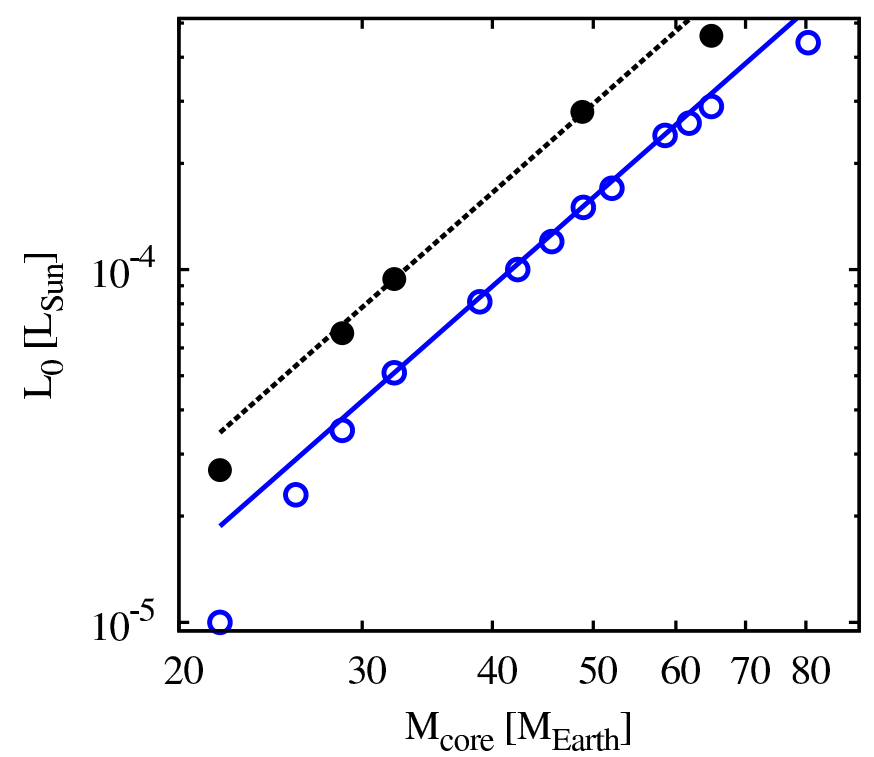

Fig. A.1. Post-formation luminosity of planets with a total mass of 5 and $10 M_{4}$ (black filled and blue open points, respectively), as a function of the mass of the core. The black dashed and blue solid lines show the corresponding power-law approximation for intermediate core masses, scaling as $M_{\text {core }}^{2.6}$.

for $10 M_{4}$ planets and core masses between 32 and $59 M_{\oplus}$. Figure A.1 shows the post-formation luminosity $L_{0}$ for planets with total masses of 5 and $10 M_{4}$ and core masses between 22 and $80 M_{\oplus}$. The lines show the corresponding power-law approximation with a scaling as $M_{\text {core }}^{2.6}$. The power-laws were normalized at the luminosity of the planet with a core mass of approximately $33 M_{\oplus}$.

We first note again the typical "luminosity inversion", i.e., the fact that for cold accretion, the luminosity directly after the end of the formation phase is lower for a more massive planet (M07), as also (partially) visible in Fig. 3. The plot further shows that the power-law approximation agrees well with the numerical results at intermediate core masses (roughly 30 to $50 M_{\oplus}$ ). For higher core masses, the increase of $L_{0}$ with increasing $M_{\text {core }}$ is in contrast weaker than predicted by the fit. For core masses less than $30 M_{\oplus}$, the numerical results also diverge from the powerlaw. At these low core masses, the dependency on $M_{\text {core }}$ is clearly stronger than $M_{\text {core }}^{2.6}$, so that the fit would predict too high $L_{0}$. This is (at least quantitatively) in agreement with the results of M07: For a $10 M_{4}$ planet with a core mass of $19 M_{\oplus}$, they find a $L_{0}$ of $2-3 \times 10^{-6} L_{\odot}$, while the power-law would predict a luminosity of $1.3 \times 10^{-5} L_{\odot}$.

\section{Appendix B: Impact of model settings on the core mass - initial entropy mapping}

As mentioned in Sect. 2, the outer boundary conditions in this work differ from the ones in Bodenheimer et al. (2000) by the additional photospheric pressure. In this section, we investigate the impact of this model setting, and additionally of the opacity due to grains in the protoplanetary envelope. The goal is to gain insight in the robustness of the core mass - initial entropy mapping within our our 1D model framework (cf. Sect. 4).

Figure B.1 shows the post-formation entropy as a function of mass. The solid lines are the same as in Fig. 2 and show the entropy for cold accretion and core masses of 32 and $49 M_{\oplus}$, and additionally for hot accretion. The dotted lines show

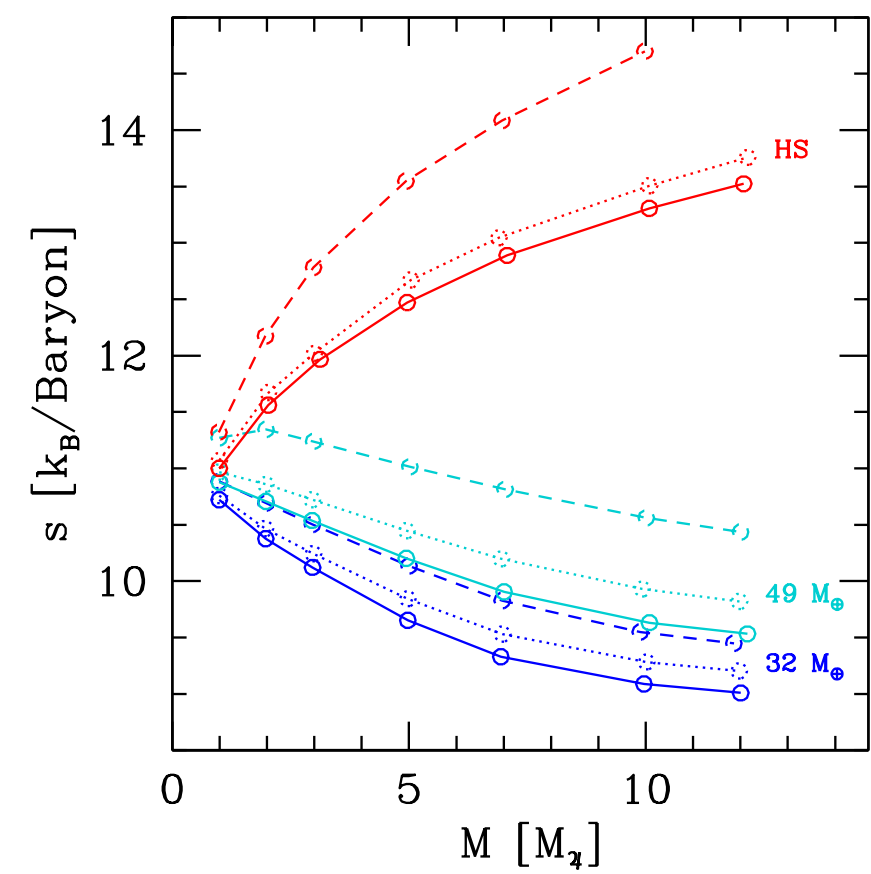

Fig. B.1. Post-formation entropy as function of total mass. The dark (light) blue lines are for cold accretion and a core mass of 32 (49) $M_{\oplus}$. The red curves are for hot accretion. The solid lines are the nominal model and identical to Fig. 2. The dotted curves are simulations without the photospheric pressure. The dashed lines are for a full ISM grain opacity.

the entropies for corresponding simulations without the photospheric pressure in the outer boundary conditions but otherwise identical settings. We see that the omission of this term leads to initial entropies that are for massive planets about 0.2 to 0.3 higher, which is a rather small change. For lower-mass planets, the impact is even lower. A generally higher entropy is expected, because with the ram pressure only, the pressure in the outermost layers is lower, while the temperature is nearly identical. The plot also makes clear that the general shape of the curves remains quite similar (for cold accretion, decrease of $S_{0}$ with $M$ and increase with $M_{\text {core }}$ ), so that the qualitative result is unchanged under a modification of these model settings.

We have also tested the impact of the opacity due to grains suspended in the protoplanetary envelope. Inspired by the work of Movshovitz et al. (2010), we have reduced the grain opacity by a factor $f_{\text {opa }}=0.003$ relative to the ISM in the nominal simulation (cf. Mordasini et al. 2012b). For comparison, M07 used a reduction factor of 0.02 . Movshovitz et al. (2010) studied the evolution of the grains during phase II of giant planet formation, therefore it is not clear if their results of can be applied to the runaway gas accretion phase of high mass planets we study here. To quantify the impact of this assumption, the dashed lines show the post-formation entropy for simulations with full ISM opacity and otherwise nominal settings. The qualitative result is the same as for the photospheric pressure: at full opacity, higher entropies result. The quantitative impact of a full ISM opacity is however greater, with difference of up to about 1.0 in $S_{0}$ (for cold accretion), which is significant. This is a consequence of the slower contraction of the radius at a higher opacity, which makes that the planet has at any given mass during the detached phase a larger radius, meaning that less gravitational potential energy is radiated at the accretion shock. Depending on the temperature, a higher grain opacity also leads to a lower photospheric pressure, which increases the entropy, as seen before. We have finally also 
found a certain inverse correlation of the distance from where the gas is assumed to fall onto the planet (infinity, $R_{\mathrm{H}}, R_{\mathrm{H}} / 3$ ) and $S_{0}$.

Qualitatively, the general correlations of $M, M_{\text {core }}$ and $S_{0}$ found in this work remain under these various changes of the model assumptions, meaning that the general trend appears to be relatively robust within our model framework. The quantitative values of the post-formation entropy are in contrast dependent in a non-negligible way on specific assumptions even within the 1D framework. Whether the general correlations persist also in $3 \mathrm{D}$ remains to be shown in future work.

\section{References}

Alibert, Y., Mordasini, C., \& Benz, W. 2004, A\&A, 417, L25

Ayliffe, B. A., \& Bate, M. R. 2009, MNRAS, 393, 49

Ayliffe, B. A., \& Bate, M. R. 2012, MNRAS, 427, 2597

Baraffe, I., Chabrier, G., Barman, T. S., Allard, F., \& Hauschildt, P. H. 2003, A\&A, 402, 701

Baraffe, I., Chabrier, G., \& Barman, T. S. 2008, A\&A, 482, 315

Baraffe, I., Chabrier, G., \& Gallardo, J. 2009, ApJ, 702, L27

Bodenheimer, P. H., \& Pollack, J. B. 1986, Icarus, 67, 391

Bodenheimer, P. H., Hubickyj, O., \& Lissauer, J. J. 2000, Icarus, 143, 2

Bodenheimer, P., D’Angelo, G., Lissauer, J. J., Fortney, J. J., \& Saumon, D. 2013, ApJ, 770, 120

Bonnefoy, M., Boccaletti, A., Lagrange, A. M., et al. 2013, A\&A, 555, A107

Burrows, A. S., Marley, M. S., Hubbard, W. B., et al. 1997, ApJ, 491, 856

Burrows, A. S., Hubeny, I., Budaj, J., \& Hubbard, W. B. 2007, ApJ, 661, 502

Chabrier, G., Baraffe, I., Leconte, J., Gallardo, J., \& Barman, T. S. 2009, AIP

Conf. Ser., 1094, 102

Chauvin, G., Lagrange, A.-M., Dumas, C., et al. 2004, A\&A, 425, L29

Commerçon, B., Audit, E., Chabrier, G., \& Chièze, J.-P. 2011, A\&A, 530, A13

D’Angelo, G., \& Lubow, S. H. 2008, ApJ, 685, 560

Deleuil, M., Bonomo, A. S., Ferraz-Mello, S., et al. 2012, A\&A, 538, A145

Fortney, J. J., Marley, M. S., Hubickyj, O., Bodenheimer, P. H., \& Lissauer, J. J.

2005, Astron. Nachr., 326, 925

Fortney, J. J., Marley, M. S., \& Barnes, J. W. 2007, ApJ, 659, 1661

Freedman, R. S., Marley, M. S., \& Lodders, K. 2008, ApJS, 174, 504

Galvagni, M., Hayfield, T., Boley, A. C., et al. 2012, MNRAS, 427, 1725

Guillot, T., Santos, N. C., Pont, F., et al. 2006, A\&A, 453, L21

Hartmann, L., Cassen, P., \& Kenyon, S. J. 1997, ApJ, 475, 770

Hubickyj, O., Bodenheimer, P. H., \& Lissauer, J. J. 2005, Icarus, 179, 415
Iaroslavitz, E., \& Podolak, M. 2007, Icarus, 187, 600

Ida, S., \& Makino, J. 1993, Icarus, 106, 210

Janson, M., Bergfors, C., Goto, M., Brandner, W., \& Lafrenière, D. 2010, ApJ, 710, L35

Konopacky, Q. M., Barman, T. S., Macintosh, B. A., \& Marois, C. 2013, Science, 339, 1398

Lafrenière, D., Jayawardhana, R., \& van Kerkwijk, M. H. 2008, ApJ, 689, L153

Lagrange, A.-M., Bonnefoy, M., Chauvin, G., et al. 2010, Science, 329, 57

Lambrechts, M., \& Johansen, A. 2012, A\&A, 544, A32

Li, S. L., Agnor, C. B., \& Lin, D. N. C. 2010, ApJ, 720, 1161

Lissauer, J. J. 1993, ARA\&A, 31, 129

Lissauer, J. J., Hubickyj, O., D’Angelo, G., \& Bodenheimer, P. H. 2009, Icarus, 199, 338

Lovelace, R. V. E., Covey, K. R., \& Lloyd, J. P. 2011, AJ, 141, 51

Marleau, G.-D., \& Cumming, A. 2013, MNRAS, submitted [arXiv: 1302.1517]

Marley, M. S., Fortney, J. J., Hubickyj, O., Bodenheimer, P. H., \& Lissauer, J. J. 2007, ApJ, 655, 541

Marois, C., Macintosh, B., Barman, T. S., et al. 2008, Science, 322, 1348

Mercer-Smith, J. A., Cameron, A. G. W., \& Epstein, R. I. 1984, ApJ, 279, 363

Miller, N. K., \& Fortney, J. J. 2011, ApJ, 736, L29

Mollière, P., \& Mordasini, C. 2012, A\&A, 547, A105

Mordasini, C., Alibert, Y., \& Benz, W. 2006, Proc. Tenth Anniversary of 51 Peg-b: Status of and prospects for hot Jupiter studies (Paris: Frontier Group), 84

Mordasini, C., Alibert, Y., Georgy, C., et al. 2012a, A\&A, 547, A112

Mordasini, C., Alibert, Y., Klahr, H., \& Henning, T. 2012b, A\&A, 547, A111

Movshovitz, N., Bodenheimer, P. H., Podolak, M., \& Lissauer, J. J. 2010, Icarus, 209,616

Ormel, C. W., \& Klahr, H. H. 2010, A\&A, 520, A43

Papaloizou, J. C. B., \& Nelson, R. P. 2005, A\&A, 433, 247

Podolak, M., Pollack, J. B., \& Reynolds, R. T. 1988, Icarus, 73, 163

Pollack, J. B., Hubickyj, O., Bodenheimer, P. H., et al. 1996, Icarus, 124, 62

Prialnik, D., \& Livio, M. 1985, MNRAS, 216, 37

Shiraishi, M., \& Ida, S. 2008, ApJ, 684, 1416

Spiegel, D. S., \& Burrows, A. S. 2012, ApJ, 745, 174

Spiegel, D. S., Burrows, A. S., \& Milsom, J. A. 2011, ApJ, 727, 57

Stahler, S. W., Shu, F. H., \& Taam, R. E. 1980, ApJ, 242, 226

Stevenson, D. J., \& Salpeter, E. E. 1977, ApJS, 35, 221

Tanigawa, T., Ohtsuki, K., \& Machida, M. N. 2012, ApJ, 747, 47

Uribe, A. L., Klahr, H., \& Henning, T. 2013, ApJ, 769, 97

Vaytet, N., Audit, E., Chabrier, G., Commerçon, B., \& Masson, J. 2012, A\&A, 543, A60

Wilson, H. F., \& Militzer, B. 2012, ApJ, 745, 54

Zapolsky, H. S., \& Salpeter, E. E. 1969, ApJ, 158, 809 